\title{
EL PARLAMENTO RAPA NUI Y SU ACTUAR INTERNACIONAL (2001-2011)
}

\section{The Rapa Nui Parliament and its International Actions (2001-2011)}

\section{NICOLÁS DELAIRE*}

Fecha de recepción: 20 de abril de 2020 - Fecha de aprobación: 5 de octubre de 2020

\section{Resumen}

Desde su creación en 2001, el Parlamento Rapa Nui introdujo una nueva forma de hacer política para hacer valer sus reivindicaciones en la isla. Una componente moderna del Parlamento fue volcarse a la elaboración de alianzas internacionales y a la participación en los foros de las Naciones Unidas. En este trabajo analizamos dicha construcción de alianzas, en particular con el Pacífico, y la intervención en las mesas de trabajo de las Naciones Unidas. Gracias a esta nueva red, y a un contexto extremadamente favorable, el Parlamento Rapa Nui pudo enrostrar con mayor peso las responsabilidades del gobierno chileno y cuestionar las instituciones políticas presentes en la isla. La finalidad de este artículo es destacar la existencia de un triángulo entre la comunidad rapanui, el Estado y las instituciones internacionales y cómo el Parlamento contribuyó a su creación y consolidación.

Palabras clave: Rapa Nui; Parlamento Rapa Nui; organizaciones internacionales.

\section{Abstract}

Since its creation in 2001, the Rapanui Parliament has introduced a new way of doing politics to assert its claims in Rapa Nui. The peculiarity of this traditional organization was to focus on the development of international alliances and participation in United Nations forums. In this paper, we analyse the construction of alliances in particular in the Pacific and the intervention of the United Nations working groups. Thank this network, and an extremely favourable context, the Rapanui Parliament was able to weigh more heavily the responsibilities of the Chilean government and question the political institutions present on the island. The purpose of this work is to highlight the existence of a triangle between the Rapanui community, the Chilean state and international institutions and how the Rapanui Parliament contributed to its creation and consolidation.

Keywords: Rapa Nui; Rapa Nui Parliament; international organization.

* Dr. en Ciencias Sociales. Investigador independiente. Artículo enmarcado en una investigación doctoral.

Correo-e: n_delaire@yahoo.fr 
Estoy en Ginebra en búsqueda de la justicia, nuestra gente fue agredida brutalmente por la fuerza armada especial de Carabineros e Investigación, en un confrontamiento desproporcional cuando hemos estado en una protesta pacífica y sin armas. $Y$ es una intimidación más dentro de nuestra trayectoria como rapanui dueños de sus tierras bajo la subyugación y ocupación del gobierno y Estado de Chile.

Estamos cansados de la ineficiencia, intransigencia y discriminación del Estado de Chile que no ha sabido cumplir el tratado entre mi reinado, nuestro rey, en 1888 hasta hoy, y el Parlamento Rapa Nui está abriendo un camino para obligar al cumplimiento de este tratado y definir el destino de mi pueblo.

(Entrevista con Erity Teave, "Rapa Nui pide autonomía", 2011)

\section{Introducción}

En los albores del siglo XXI, el recién configurado Parlamento, heredero ${ }^{1}$ del Consejo de Ancianos $\mathrm{N}^{\circ} 2^{2}$, redibujó una nueva forma de hacer política en Rapa Nuil ${ }^{3}$. En su inscripción notarial del mes de agosto de 2001 se estipulaba lo siguiente:

\section{Las constantes irregularidades en las adquisiciones de estas tierras debido en primer lugar a la inexistencia de una normativa justa y reconocedora de los derechos autóctonos y en segundo lugar a la actuación de una autoridad sometida a los límites del Estado como país colonizador hacen necesaria la creación e interven- ción de este Parlamento, al objeto de que la población nativa pueda conservar su legítima propiedad. (Escri- tura pública de la creación de un movimiento social denominado Parlamento Rapa Nui, 13 de agosto de 2001, Hanga Roa, APRN, 6765-6780)}

Las peticiones enunciadas en esta inscripción no dejan entrever las nuevas estrategias que iban a elaborar sus miembros algunos años después y que le dieron un protagonismo insos- pechado al Parlamento y sus reivindicaciones. Basándose en los precedentes históricos de Rapa Nui, esta entidad buscó crear y establecer alianzas estratégicas con los diferentes organismos de representación autóctonos del Pacífico Sur. Aprovechando un contexto favorable para las reivindicaciones indígenas, cristalizado en la Declaración de las Naciones Unidas sobre los Derechos de los Pueblos Indígenas en 2007, el Parlamento Rapa Nui se inscribió en una tendencia globalizante, en el marco de la cual amplió su retórica anticolonial y sus lugares de intervención. La question rapanui entraba en una nueva configuración, declinada en tres vértices: comunidad, Estado y dimensión internacional ${ }^{4}$. Nuestra propuesta en este artículo es evidenciar y analizar el rol preponderante del Parlamento Rapa Nui en este esquema en cuanto actor clave en la internacionalización del conflicto en la primera década del siglo XXI. Se presentarán aquí dos ejes internacionales en los que el Parlamento trabajó de manera sostenida: el primero ligado al mundo del Pacífico sur y el segundo a las diferentes organizaciones relativas a esta área en las Naciones Unidas.

La metodología empleada en este trabajo fue la revisión de diversas fuentes nacionales e internacionales. Los documentos consultados fueron: las comunicaciones emitidas por el Parlamento Rapa Nui, fuentes provenientes de la Gobernación regional, del Ministerio de Relaciones Exteriores y de las Naciones Unidas y artículos de la prensa nacional, principalmente entre 1990 y 2015 . El enfoque teórico utilizado para la lectura de los archivos es el del análisis crítico del discurso (ACD) de Teun van Dijk (1999). A partir de esta apuesta analítica se buscó reconstruir diversas situaciones coloniales en las que se pueden destacar los 
hilos de la genealogía del poder en Rapa Nui. Finalmente, es vital destacar el concepto de dialogismo utilizado por Mijaíl Bajtín (2005), que resulta clave para rescatar las múltiples voces presentes en una dinámica colonial.

\section{La construcción de un espacio del Pacífico conjunto}

De manera sucinta, se puede mencionar que los pueblos polinésicos tuvieron dos causas primordiales de movilización a partir de la segunda mitad del siglo XX. La primera estuvo ligada al escenario militar, pues las principales potencias occidentales usaron los diferentes archipiélagos como sitios de pruebas nucleares (desde 1946, por parte de Estados Unidos, hasta 1996, por parte de Francia). Frente a los riesgos de las pruebas atómicas, Fidji y Nueva Zelanda comenzaron a organizarse de manera sistemática a partir de la década de 1970. Como consecuencia, la Asamblea General de la ONU aprobó, en la Resolución 3.477, la creación de una zona libre de armas nucleares en el Pacífico meridional, abogando, además, por una cooperación de los países que poseyeran armas nucleares. En el Foro del Pacífico Sur, en Rarotonga, en las islas Cook, se aprobó, en agosto de 1985, el Tratado de Zonas Libres de Armas Nucleares del Pacífico Sur.

El segundo punto de movilización se orientó a la descolonización de los diferentes archipiélagos y la inscripción de algunos de estos en la lista de los territorios autónomos de las Naciones Unidas. Los pueblos originarios de esta región, víctimas de violencias coloniales con tintes nucleares, no solo se respaldaron en el apoyo de las organizaciones internacionales, también crearon mecanismos de lucha desde sus propias agendas.
Linda Tuhiwai Smith (2012) entrega luces respecto de los movimientos de los pueblos originarios del Pacífico. Señala que estos articularon sus reivindicaciones en torno a cuatro ejes: la descolonización, la sanación, la transformación y la movilización. Estos cuatro puntos no eran solo objetivos, sino que procesos conjuntos que informaban y clarificaban las tensiones locales, regionales y globales (ídem, p. 120). Con el paso del tiempo, ahonda Smith, "los diferentes movimientos han desarrollado y compartido un discurso o lenguaje internacional que permite a los activistas indígenas hablar entre ellos más allá de sus diferencias culturales" (ídem, p. 114). Cabe mencionar que, en el caso de la Polinesia, se produjo progresivamente, entre todos los archipiélagos, un sentimiento identitario y una historia común. En ese sentido, Rapa Nui, por cercanía e historia compartida, siempre tuvo como referente cercano a Tahiti. La circulación del saber se fue incrementando con el tiempo y se convirtió en una fuente de inspiración y emulación para los líderes del Parlamento.

Ya en el año 2004, el Parlamento mostraba una voluntad real de establecer lazos y nexos con los archipiélagos vecinos. En el reporte regional del Pacífico presentado por la motarilavoa Hilda Lini ${ }^{5}$, directora del Pacific Concerns Resources Centre (PCRC), se estableció una nueva carta magna de los países de la región. En esta declaración, la directora definía orígenes históricos comunes de los pueblos del Pacífico, a saber: guardianes del océano Pacífico; protectores del medioambiente y la biodiversidad; poseedores de una herencia cultural y de recursos naturales, y guardianes del conocimiento de los pueblos originarios. Por otra parte, identificaba tres categorías del mundo indígena en la región: Rapa Nui entraba en la de "Pueblos indígenas viviendo 
bajo administración colonial, invasión, ocupación, integración y asimilación”6.

Según la misma interventora, estos últimos pueblos sufrían el colonialismo, la imposición de las culturas extranjeras y una educación occidental. Esta situación ponía en evidencia los diferentes abusos sufridos por los indígenas del Pacífico y, sobre todo, según las Resoluciones № 15/14 de 1960, una clara falla del rol político de las Naciones Unidas. En este balance, la expositora subrayaba que el Pacífico, como también el Caribe, eran regiones donde quedaba una importante cantidad de territorios colonizados. Estos pueblos subyugados habían apelado a la Comisión de Derechos Humanos y al Foro Permanente de las Naciones Unidas. De esta forma, el PCRC proponía las siguientes recomendaciones:

1. Enviar un relator especial para visitar la situación de los derechos fundamentales y las libertades de los pueblos indígenas. Ver las violaciones infligidas por las administraciones coloniales.

2. Iniciar un diálogo con el reportador del comité de descolonización y ver la necesidad de reinscribir territorios del Pacífico en la lista de 1963, como Papúa Occidental, Polinesia Francesa, Maluku, Ka Pae'aina y Rapa Nui.

3. Pedir un reporte que comprendiera un verdadero análisis de los pueblos indígenas que viven bajo ocupación colonial y en autodeterminación, como en el caso de Papúa Occidental, Te Ao Maohi, Ka Pae'aina, Maluku, Rapa Nui, Aotearoa, aborígenes de Australia, Islas Torres, Bougainville, Wallis y Futuna y las islas Mariana?.
La síntesis establecida por Lini permitía que los otros pueblos concernidos en este informe pudieran hacer valer sus voces. En estas mismas fechas, el Parlamento emitió dos documentos claves para entender esta nueva dimensión internacional, en los que las conexiones con este reporte son evidentes. En primer lugar, el Parlamento integraba el aspecto jurídico internacional con el cual los pueblos polinésicos habían obtenido un cierto éxito. La idea de autonomía ya estaba anclada en la visión de la institución rapanui, así como la potencial desaparición de su cultura. Esto puede apreciarse en la documentación entregada a la Gobernación el 3 de noviembre de $2003^{8}$.

En segundo lugar, se buscaba establecer una conexión real con los otros pueblos polinésicos que pudiese subsistir en el tiempo. Dichas alianzas eran de orden económico, jurídico y político, y buscaban crear una zona autónoma del Pacífico ${ }^{9}$. Desde un punto de vista internacional, el Parlamento apelaba a una unión entre las diferentes organizaciones de la zona. Esta unificación de los pueblos polinésicos demostraba una clara elaboración identitaria regional, en la que se reconocían patrones e historias comunes. Friedman (2003) propone una definición de regionalización como "la reidentificación de las áreas subnacionales como grupos culturalmente unificados, que exigen derechos culturales y políticos, los cuales a veces incluyen soberanía" (p. 747). Por su parte, Muñoz (2017) señala que, para los rapanui, Tahiti era y sigue siendo sinónimo de riqueza y prosperidad debido a una conexión temporal que remonta a la venida de los misioneros católicos franceses en el siglo XIX. El imaginario cultural y político rapanui es tributario de la Polinesia francesa. 
Si se analiza este manifiesto bajo un prisma nacional, destaca en él la denuncia de la dominación política y económica que establecía un marco colonial por parte del Estado chileno. Este expandía sus redes dentro de la comunidad, donde establecía lazos con las autoridades locales. La corrupción era un factor clave para entender la división de intereses entre los diferentes grupos originarios. La necesidad de una autonomía era entorpecida, de este modo, por el Estado, en dos tiempos. Por un lado, establecía una relación de dominación, mediante las diferentes instituciones en la isla, con organizaciones intermediarias. Por el otro, los intereses económicos privilegiaban algunos grupos, que eran ratificados en los puestos de poder. Es por esta razón, y según esta lectura, que el Parlamento Rapa Nui apelaba a nuevos actores que tuviesen un patrón histórico común y objetivos similares. En otras palabras, era desde la tradición rapanui, pero también polinésica, que el Parlamento podía rechazar las instituciones emanantes del Estado, la Comisión de Desarrollo de Isla de Pascua (CODEIPA), sus representantes, el gobernador y el alcalde, y su marco legal, la Ley Pascua y la Ley 19.253. Según su construcción histórica, el Parlamento ya existía antes de la firma del Acuerdo de 1888, lo que permitía desmarcarse del Estado dibujando una autonomía previa e impostergable.

En 2007, el Parlamento envió un documento a Rex Rumakiek, director asistente de la oficina de Descolonización del PCRC en Suva (Fiyi) ${ }^{10}$. Este documento es extremadamente valioso pues da indicios sobre las participaciones anteriores del Parlamento y de su predecesor, el Consejo de Ancianos $\mathrm{N}^{\circ} 2$. Establece, además, una síntesis de los dos puntos relativos a las reivindicaciones en el Pacífico esbozadas anteriormente. En la carta se subraya que ya el 21 de septiembre de 1999 el Consejo de Ancianos № 2 había participado en la conferencia del Nuclear Free and Independent Pacific (NFIP) en Arue, Tahiti. En esa ocasión, sus emisarios habían denunciado los abusos del gobierno chileno hacia los rapanui. El Consejo fue reconocido como miembro del NFIP y durante la conferencia se recomendó la inscripción de Rapa Nui en la lista de los territorios no autónomos. En otras palabras, esta última fue vista como un territorio que debía ser descolonizado. En el mismo documento enviado al PCRC, se destacaba la participación del Parlamento en 2003 en la conferencia de la NFIP en Tonga, donde fue reafirmada la petición de descolonización. Para llevar a cabo esta solicitud, el Parlamento contó con el apoyo de Lorenz Gonschor, quien coordinó los esfuerzos de descolonización en los archipiélagos del Pacífico. Para el Parlamento, el objetivo era establecer un grupo de trabajo similar a los existentes en Papúa Occidental, Nueva Caledonia y Tahiti, los cuales estaban demandando ya a la descolonización en las Naciones Unidas.

Es en este contexto que el Parlamento recibió una copia de las propuestas del PCRC para la mesa de actividades ligada a la descolonización para los años 2007-2009. En la carta antes citada, el Parlamento retomaba la Resolución 1.541 de 1960 para demostrar que Rapa Nui calificaba ampliamente como territorio no autónomo. Según los mismos autores, Chile había impuesto y forzado su integración aprovechándose del aislamiento geográfico y de una cultura diferente. Esta secuencia había generado, en el tiempo, efectos devastadores en los ámbitos cultural, social y económico. El Parlamento finalizaba este documento recalcando que "El pueblo rapanui desea tener la 
[elección] entre la independencia o la libre asociación como futuro estatuto político"11.

Progresivamente, el Parlamento empezó a controlar la discursividad de esta lucha en un marco internacional donde el gobierno chileno fue continuamente emplazado. Un ejemplo de esta situación fue la carta del Parlamento del 19 de mayo de 2008, en la que se denunciaban las tensiones entre el Estado y la comunidad. En esta, la vocera del Parlamento explicaba que habían agotado los medios (instancias de diálogos y acuerdos con el gobierno de Chile) y hacía un recuento de la historia de opresión infligida al pueblo rapanui. Precisaba que en 2002 se conoció el texto original del Convenio (Acuerdo), en el que se enfatizaba que Atamu Tekena jamás había entregado la soberanía ${ }^{12}$. En esa misma fecha, el Parlamento presentó un proyecto de autodeterminación. Frente a estas iniciativas, Chile demoró este requerimiento y confió el poder al gobernador en desmedro de las instituciones locales. Como consecuencia de esta indolencia, se pedía a Chile abandonar el territorio insular para que, de esta forma, pudiera convertirse en un país independiente. Esta carta, dirigida a la presidenta Michelle Bachelet, fue enviada con copia a Gabriel Teri'i Tahi, presidente de la Pacific Islands Association of Non-governmental Organisations (PIANGO) $)^{13}$.

Mientras se enviaban estas misivas a instituciones internacionales, el Parlamento se movilizó, a su vez, en la isla. La situación expuesta demuestra cómo se fue tejiendo la triangulación del conflicto entre las organizaciones internacionales, el Estado y la comunidad rapanui. Esta idea puede ser ilustrada con la manifestación realizada en mayo de $2008^{14}$. Tras dejar constatación de su postura en PIANGO, el Parlamento reafirmó su voluntad política, ya expresada en los foros, durante las manifestaciones de los días 20 y 21 de mayo en la isla. El motivo era "proclamar nuestra posición en relación al Estatuto Especial de Administración para Rapa Nui y afirmar nuestro Derecho a la autodeterminación"15. Vale detenerse en esta estrategia, pues nos deja entrever la manera en que el Parlamento actuó en los diversos ámbitos, lo cual será una tendencia y una característica de su accionar hasta el año 2015.

\section{Participación en los foros de la ONU}

Una nueva ventana de participación internacional para el Parlamento Rapa Nui fueron los foros de las Naciones Unidas. El Parlamento prosiguió con su dinámica política buscando otros actores que tuviesen un patrón histórico común y objetivos similares. Estos hicieron un frente común en la Tercera Sesión del Foro Permanente para las Cuestiones Indígenas de la $\mathrm{ONU}^{16}$, acaecido entre el 10 y el 21 de mayo de 2004 en Nueva York. Cabe destacar la relevancia de las denuncias emitidas por el Parlamento en esta instancia, en las que se enjuiciaban las acciones del Estado chileno en la isla. Estas eran expuestas de la manera siguiente:

1. Rapa Nui necesita un Relator de Derechos Humanos para Rapa Nui, Te Ao Maohi, Hawaii, Papua Occidental y Maluku, para constatar los actos terroristas cometidos por el Gobierno Chileno quien destruyó las casas de dos familias el 16 de Julio del año 2001.

2. Pedimos al Foro Permanente que asesore al Comité de Descolonización de la ONU para que inscriba Rapa Nui, Te Ao Maohi, Hawaii, Papua Occidental y Maluku, en la lista de territorios no autónomos. 
3. El Parlamento Rapa Nui pide al Relator Especial Doctor Stavenhagen que el Estatuto de Autonomía para Rapa Nui sea preparado en Rapa Nui, con la participación del Parlamento y del Consejo de Monumentos Kahu Kahu Ohera y Tu'u Hotu Iti.

4. Que los Estados revisen sus Constituciones y Legislaciones para garantizar a los pueblos originarios sus derechos [...].

5. Rapa Nui pide al Foro Permanente y a la Comisión de Derechos Humanos abordar el tema de la Soberanía Rapa Nui que no ha sido evaluada ni debatida.

6. El Gobierno de Chile considera que Rapa Nui ha sido integrada, sin embargo, esto no ha sido cierto [...] no se le ha proporcionado al pueblo el derecho político completo debido a la integración, como se establece en la Resolución 15-14 y 1541 de la Asamblea General de la ONU.

7. Rapa Nui pide al Foro Permanente aconsejar al Gobierno de la República de Chile para evitar los obstáculos políticos relativos a la descolonización de Rapa Nui ${ }^{17}$.

Las peticiones expuestas por el Parlamento estaban directamente relacionadas con la visita del relator especial de la ONU, Rodolfo Stavenhagen, en julio de 2003, y con su informe entregado en noviembre del mismo año. Si bien el relator no pudo ir a Rapa Nui, sí se reunió con el alcalde y miembros del Consejo de Ancianos № 1, ocasión en que se le señaló los problemas relativos a la adquisición de tierras por parte de chilenos que no eran necesariamente rapanui. En esta reunión, los representantes rapanui reclamaban ya por el tema de la migración que hacía peligrar su identidad cultural. Sobre estos encuentros debe precisarse que no causaban unanimidad en el seno de la comunidad. En la revista Rongo Rapa Nui, editada por la Gobernación, se comentaba la visita de Stavenhagen enfatizando dos puntos: 1) el "drama de la tierra es un conflicto resuelto en la isla, no así para las etnias americanas" y 2) "la prensa continuará difundiendo los proyectos independentistas que han sido desplazados en cada elección realizada en los últimos años en la Isla denostando a los afanes independentistas" (Rongo Rapa Nui, 2003, p. 1). Estas opiniones mostraban ya la tónica de la isla desde la separación del Consejo de Ancianos en 1994 y las opiniones divergentes entre ambos grupos. Las tensiones fueron aumentando cada vez más producto de la importancia creciente del Parlamento en las diversas tribunas internacionales.

El balance de la primera visita del relator de la ONU a Chile puso en evidencia las dificultades del Estado respecto de las políticas indígenas. Pese a reconocer la buena voluntad de la Concertación de Partidos por la Democracia, que se desmarcó de la dictadura mediante la promulgación de la Ley 19.253, las conclusiones no fueron alentadoras para Chile. En un inicio, Stavenhagen hacía hincapié en que Chile adoptase el Convenio 169 de la Organización Internacional del Trabajo (OIT) y otros convenios internacionales que garantizaban los derechos humanos de los indígenas. Posteriormente, el Estado chileno respondió a estas demandas señalando que las modificaciones de la Ley 19.253 eran imposibilitadas por el Congreso, que rechazaba todo tipo de reforma. Para este efecto, el relator pedía una agilización del proceso de la reforma constitucional. Dentro de estos cambios, agregaba, era crucial que se ampliase el Fondo de Tierras, manejado 
por la Corporación Nacional de Desarrollo Indígena (CONADI), para la entrega de las propiedades a los pueblos originarios. Para el caso de Rapa Nui, Stavenhagen afirmaba, en sus conclusiones, que "El proyectado estatuto de autonomía de la Isla de Pascua (Rapa Nui) deberá contener garantías de protección de los derechos del pueblo originario Rapa Nui sobre sus tierras, recursos y el respeto a su organización social y vida cultural” (2003, p. 19).

Dentro de un contexto cada vez más favorable para los derechos de los pueblos originarios, simbolizado en la declaración del 13 de septiembre de 2007, el Parlamento volvió nuevamente a la tribuna en el mes de abril de $2008^{18}$, durante la Séptima Sesión del Foro Permanente de la ONU para las Cuestiones Indígenas. En esta presentación, la vocera del Parlamento se sumaba a Santi Hitorangi, representante del clan Hito. La argumentación del Parlamento abogaba en el sentido de la temática de la mesa de ese año ${ }^{19}$. Por una parte, la vocera subrayaba que: "Descolonización y libre determinación son los objetivos más significativos de nuestros derechos humanos para erradicar el colonialismo, privaciones y otras violaciones"20. Para preservar su cultura e integridad, el Parlamento insistía en su deseo inherente de libre determinación producto de la actitud colonialista del Estado de Chile. Dicha actitud amenazaba la cultura rapanui con una probable extinción debido al poco cuidado del Estado. Esta extinción se debía al deterioro y el colapso de la isla, segundo argumento que permitía entender la necesidad de fijar una regulación migratoria. Por otra parte, el Parlamento pedía ser integrado al Seminario de Descolonización encabezado por Carlyle Corbin, así como también a la lista de los territorios no autónomos. En la conclusión de esta presentación, los representantes rapanui pedían que la ONU y sus agencias respectivas usasen la reciente Declaración de los Derechos de los Pueblos Indígenas. Esta petición era imperativa para encontrar una salida real a las necesidades de los pueblos originarios.

Es en esta dinámica que, en el mes de abril de 2009, el relator de las Naciones Unidas, James Anaya, inició una visita a Chile. Estas visitas solo podían dar mayor fuerza a los planteamientos indígenas. Cabe recordar que el Estado chileno había ratificado, un año antes, el Convenio 169 de la OIT. Durante esa misión, los objetivos de Anaya eran ver las ejecuciones de las propuestas de su antecesor, a saber: el reconocimiento de los derechos de los pueblos originarios y las reformas constitucionales del Estado. El relator especial, en un comunicado del 9 abril de 2009, hizo un llamado a que:

\footnotetext{
Se lleve a cabo un proceso de consulta sobre la reforma constitucional que sea conforme a las normas internacionales aplicables, incluidas aquellas reconocidas en el Convenio № 169 y en la Declaración de las Naciones Unidas sobre los derechos de los pueblos indígenas, y que el resultado de la reforma también sea conforme a estas normas. (Anaya, 2009, p. 22)
}

Este llamado se sumaba a los convenios firmados por Chile y a las dudas que habían provocado entre los representantes chilenos en la ONU. El relator volvía a recordar al gobierno la serie de obligaciones que les competía respecto de los pueblos indígenas, como "la adopción de medidas legislativas, administrativas o políticas que afecten directamente sus derechos y sus intereses" (Anaya, 2009, p. 32).

Estas recomendaciones dieron mayor fuerza a los movimientos indígenas durante el Foro de 2009. En la Octava Sesión, que tuvo lugar entre el 18 y el 29 de mayo, en la ONU, el Parla- 
mento pudo nuevamente pronunciarse en la mesa relativa a los derechos humanos. En un trabajo conjunto con otros pueblos originarios que vivían bajo dominación colonial, la vocera del Parlamento presentó las siguientes ideas ${ }^{21}$.

En primer lugar, expuso que existía la "obligación sagrada" de permitir la autodeterminación y el autogobierno a muchos pueblos indígenas que residen en el Pacífico y el Caribe. Estos territorios habían sido ubicados por la ONU en la lista de los territorios no autónomos. Algunos Estados dominantes habían adherido a estas demandas, pero otros habían removido, de manera unilateral, los territorios a descolonizar. La vocera del Parlameto agregó, en segundo lugar, que en el caso de Rapa Nui y Maluku, signatarios de esa intervención, habían surgido problemas de descolonización. En tercer lugar, precisó que su presentación buscaba denunciar la demora de estos procesos y las trabas interpuestas por los países colonizadores.

Uno de los argumentos claves era que, a pesar de las denuncias de racismo apoyadas por el Comité para la Eliminación de la Discriminación Racial (CERD en inglés), el Comité de Descolonización había rechazado responder a las diferentes demandas. Frente a estas demoras, la mesa encabezada por la vocera del Parlamento Rapa Nui ilustraba cómo, durante diez años, la Comunidad de los Estados Caribeños (CARICOM en inglés) había denunciado estas situaciones sin consecuencias mayores. El malestar sobre este disfuncionamiento era tal que se llegó a acusar de ejercer una "política de acomodación colonial" a los territorios colonizadores. A modo de solución, los miembros de las organizaciones indígenas buscaron el apoyo del doctor Corbin. En su informe, este recordaba que el Foro debía encabezar y demostrar la necesidad de que se tomasen las medidas correspondientes a la Declaración de las Naciones Unidas sobre los Derechos de los Pueblos Indígenas (DNUDPI), que incluían a los que vivían en territorios no autónomos.

Esta presentación tuvo ecos importantes para los planteamientos del Parlamento. Esto puede verse en las cartas enviadas a la presidenta Bachelet, el 30 de julio de ese mismo año, y al subsecretario del Interior, Patricio Rosende, el 21 de octubre. Al detenerse en los puntos esbozados, la primera noción expuesta tenía que ver con la autonomía para los pueblos originarios. El debate de estos años cobró aún más fuerza tras esta declaración, pues se establecía un lazo de solidaridad con otros pueblos en la misma situación. Sin embargo, los nuevos estatutos propuestos por el Estado chileno no cumplían con lo establecido en las recomendaciones de Corbin y con los objetivos del Foro de Descolonización. En la lectura del Parlamento, este desistimiento era la clara demostración de la permanencia de una actitud colonizadora iniciada desde 1888. Al dirigirse a la presidenta Bachelet, la organización rapanui señalaba que:

\section{En la misma Constitución chilena, artículo 19 n8 se señala el derecho a vivir en un medio ambiente libre de contaminación y en el mismo artículo 19 nำ7 la Consti- tución chilena se asegura a todas las personas el dere- cho a la libertad personal y a la seguridad personal en consecuencia ${ }^{22}$.}

Desde la firma del Acuerdo de 1888, a juicio del Parlamento, el gobierno chileno nunca cumplió su deber de proteger a los rapanui. Por esta razón se podía entender la voluntad de querer inscribir a la isla como nuevo territorio a descolonizar. Junto al problema de la autonomía de Rapa Nui, se presentaba conjuntamente el riesgo y el peligro que corría la cultura rapanui. 
El argumento esbozado era el de la migración elevada y descontrolada hacia a la isla. En la carta enviada al subsecretario Rosende, el Parlamento recalcaba:

\begin{abstract}
Al iniciar la problemática del tema inmigración, es decir el ingreso y permanencia de chilenos continentales en cantidades masivas al territorio de la isla [...] provocando como resultado inmediato la contaminación ambiental, el desempleo masivo, la sobreexplotación de recursos, la aculturación y la transculturación, entre otros efectos negativos ${ }^{23}$.
\end{abstract}

Si se ahonda en la presentación aludida en el Foro Permanente, se constata una parte relevante de la base discursiva del Parlamento ligada a la agenda de descolonización. Esta idea podía asociarse a lo que el Parlamento hacía saber a la presidenta Bachelet: "Las Naciones Unidas han señalado que el año 2010 se pone término a la descolonización y desmilitarización"24. Con este argumento, la lógica independentista propuesta por los rapanui cobraba toda su fuerza al buscar protegerse del incumplimiento del Estado. En este sentido, el Parlamento se desmarcaba de las observaciones optimistas del relator Anaya. En la misiva enviada al subsecretario Rosende se ofrecían nuevos datos con respecto de la organización propia de los indígenas sin tener que pasar por un conducto oficial. La carta detallaba lo siguiente:

\footnotetext{
El Parlamento Rapa Nui respaldado por la comunidad Rapa Nui y sus familias y por corporaciones, asociaciones, agrupaciones y otras organizaciones comunitarias Rapa Nui, totalmente independientes del Estado en vista de la insistente falta de voluntad política ha resuelto desplegar una manifestación masiva y pacífica en oposición a todas las medidas ya aludidas próximamente ${ }^{25}$.
}

Este primer momento discursivo, puntuado por manifestaciones pacíficas en la isla $y$ alianzas internacionales, fue acelerándose con el gobierno de Sebastián Piñera. Las tensiones y los abusos de los años 2010 y 2011 dieron una fuerza aún mayor al Parlamento y legitimaron su presencia en los escenarios internacionales. Este período brindó una cantidad mayor de documentos, intercambios de cartas y presentaciones en los diversos foros internacionales. Los actos violentos del gobierno, ampliamente difundidos por los diferentes medios de comunicación, evidenciaron, en las diversas escalas local, nacional e internacional, la tensión colonial existente entre los rapanui y la metrópoli. Frente a organizaciones desprestigiadas o cercanas al Estado, el Parlamento pudo afirmarse en primera línea.

Un mes después del cambio de mando, el Parlamento Rapa Nui se dirigió al presidente Piñera. Los objetivos de esta carta gravitaron, en primer lugar, en torno al proyecto de Control de Ingreso y Permanencia de personas nacionales y extranjeras en el territorio de Isla de Pascua. Un segundo elemento discursivo presente en esa carta fue la confirmación de la postura de esta organización frente a la descolonización:

\begin{abstract}
Considerarse la aclaración de las Naciones Unidas Resolución 1.514 sobre concesión de Independencia de países y pueblos colonizados, decimoquinto periodo de sesiones de la organización año 1960 proclamando solemnemente la necesidad de poner fin rápida e incondicionalmente al colonialismo en todas sus formas y manifestaciones y a dicho efecto se declara en su punto $1,2,3$ y $4^{26}$.
\end{abstract}

Tal como puede apreciarse, el Parlamento Rapa Nui proseguía con la agenda internacional relativa a los planteamientos regidos por el Comité de Descolonización. Como fue expuesto anteriormente, las tensiones siguieron aumentando en la isla, razón por la cual Rafael Tuki (consejero rapanui en CONADI) manifestó 
su descontento a Carlos Llancaqueo, comisionado presidencial para Isla de Pascua. En esta carta, Tuki emplazaba al presidente Piñera por su mutismo frente a las misivas enviadas en las que se exponían las temáticas citadas. La argumentación del consejero era notable, pues evidenciaba la falta de compromiso del Estado. Esta primera idea recordaba el argumento ya conocido del cuasi abandono de la isla por parte de Chile. Esta desatención era aún más notoria pues se omitían las violencias y los abusos policiales, a propósito de lo cual Tuki subrayaba la noción de genocidio silencioso. Proseguía, en esta vía, indicando que:

\begin{abstract}
El Presidente tiene la oportunidad histórica de revertir esto y transformar la relación de nuestros pueblos en base a justicia y dignidad, respetando nuestro derecho inalienable a la Libre Determinación, derechos que están garantizados en la letra del Tratado de Acuerdos de Voluntades que celebró nuestro pueblo con Chile en 1888, en la Declaración de la ONU sobre los Derechos de los Pueblos Indígenas, en los Pactos Internacionales de Derechos Humanos, la Convención sobre la Discriminación Racial, Convenio 169 de la OIT y la Resolución 1514-1541 de la ONU²7.
\end{abstract}

Tuki veía con lucidez que urgía el reconocimiento de las organizaciones políticas tradicionales rapanui al tenor del derecho internacional moderno. Cuidadosamente, el autor prefería evitar el término de independencia, pues lo que imperaba era avanzar hacia una autonomía con organizaciones locales validadas por la misma comunidad. Esta propuesta obedecía a un compromiso que había sido establecido, tiempo atrás, por el presidente Ricardo Lagos. El mensaje concordaba, en su casi totalidad, con los planteamientos del Parlamento adquiridos en sus continuas presentaciones internacionales. Tuki los legitimaba reconociéndolos como una organización tradicional y válida a la hora de reivindicar los derechos de la comunidad.

El Parlamento ya había establecido, en cartas anteriores, que la única solución existente era la independencia, lo que produjo una tensión mayor con el Estado. Estas fueron incrementándose con el pasar de los meses, como lo atestigua la documentación que analizaremos a continuación. La actitud del gobierno de Piñera siguió produciendo el rechazo de gran parte de la comunidad. En una misiva del 30 de agosto al Ejecutivo, la institución rapanui reafirmaba su principio de representatividad ${ }^{28}$. Este argumento había sido ya esbozado en las recomendaciones del relator Anaya, en las que se indicaba que se debía privilegiar a las organizaciones indígenas. Por otra parte, los emisores postulaban el fin del colonialismo en la isla y volver a ser lo que eran previo a la llegada del Estado chileno a Rapa Nui.

\section{Violencia y represión del Estado y las acciones del Parlamento Rapa Nui}

En esa misma fecha, numerosos clanes optaron por recuperar sus espacios tradicionales, pertenecientes ahora a instituciones chilenas. La mayoría de estas ocupaciones fueron desalojadas violentamente por las fuerzas especiales de Carabineros. Uno de estos lugares "recuperados" correspondía al hotel Hangaroa, propiedad de la familia Schiess, terreno reclamado por el clan Hitorangi (Delsing, 2017). Este espacio fue ocupado por hombres, mujeres y niños, quienes se tomaron en diversas ocasiones el hotel. Las respuestas de las fuerzas policiales fueron desmedidas y provocaron una oleada de críticas al rol del Estado en la isla. 
Es en este tenso clima policial reinante que el 20 de noviembre de 2010 el Parlamento volvió a comunicarse con el poder ejecutivo ${ }^{29}$. El manejo de las discusiones en los foros internacionales más el apoyo de diversas ONG demostraron que el Parlamento se movía con habilidad en los múltiples escenarios. El presidente chileno brindó el mismo año un discurso en las Naciones Unidas. En la Asamblea General, el 23 de septiembre, el mandatario declaró: "Nosotros nos sentimos muy orgullosos de ser una nación multicultural, pero reconocemos que por siglos no hemos dado a nuestros pueblos originarios las verdaderas oportunidades que ellos merecen y necesitan" (Cooperativa, 2010a).

Es desde este argumento que el Parlamento Rapa Nui emplazó al Ejecutivo al denunciar incumplimientos y dilatación por parte del gobierno, lo que entraba en contradicción con la Declaración de los Pueblos en su libre determinación. Para hacer frente a estas faltas, la institución isleña, en el nombre de la "Nación Rapa Nui", se veía obligada a luchar por la reivindicación del territorio de acuerdo con la Resolución 1.514-1.541 de la Carta de la Asamblea General de las Naciones Unidas.

La violencia policial ligada al desalojo del 3 de diciembre de 2010 movilizó la opinión pública nacional e internacional. Esto trajo como consecuencia directa, por ejemplo, un informe de la Asociación Latinoamericana para los Derechos Humanos (ALDHU). En ese balance, ofrecido por su presidente Juan Pablo Letelier, se sostenía que: "El Estado chileno está obligado a agotar todas las vías del diálogo y la resolución pacífica de cualquier conflicto que afecte derechos de estos pueblos originarios" (2010, p. 10). Durante la reunión encabezada por Letelier con miembros del Parlamento Rapa Nui, estos indicaron que estaban siendo asesorados por abogados del Indian Law Resource Center para llevar la información a la Comisión Interamericana de Derechos Humanos (CIDH).

Dentro de este informe, Letelier solicitaba la visita de James Anaya, relator especial de las Naciones Unidas para los Pueblos Indígenas, y pedía la adopción de medidas cautelares en la $\mathrm{CIDH}$. Además de buscar el amparo de la causa rapanui, Letelier incluyó, en el reporte, una nota enviada al ministro del Interior, Rodrigo Hinzpeter, el 3 de diciembre, en la que afirmaba que "La situación descrita afecta los derechos de los pueblos originarios y debe ser resuelta en cumplimiento con las normas del Convenio 169 de la OIT, que como Ud. sabe, son obligatorias para Chile" (Letelier, 2010, p. 44). Leonardo Crippa, miembro del CIDH y abogado representante de los 29 clanes rapanui, señaló, el 7 de diciembre: "A pesar de la solicitud de información de la Comisión Interamericana [...] hasta el día de hoy Chile no ha presentado información alguna, ha guardado absoluto silencio ante la comunidad internacional, a pesar de este pedido formal de la Comisión Interamericana" (Cooperativa, 2010b).

Los eventos del 3 de diciembre aceleraron la intervención de los diferentes organismos internacionales y el Estado tuvo que dar respuestas. La comunicación de James Anaya, en particular, movilizó a la Misión Chilena en Ginebra (Anaya, 2011). En su declaración al Ejecutivo del 9 de diciembre, el relator especial daba cuenta de la situación y pedía clarificar los hechos relativos a los actos de violencia producto del desalojo. Anaya partía reconociendo que Chile había tenido buena voluntad, destacaba el compromiso firmado el 6 de agosto entre los representantes del Estado y los grupos de trabajo para la isla, relativo a la revisión de los siguientes 
puntos: 1) La situación de tierras en la isla; 2) El estatuto administrativo especial para la isla; 3) El estatuto legal que regule la migración; 4) La elaboración de un plan de desarrollo integral.

Sin embargo, pedía que se le proporcionase información sobre las medidas tomadas y que se sancionase a los responsables. Frente a estas peticiones, el representante permanente de Chile ante la Oficina de las Naciones Unidas en Ginebra, Pedro Oyarce, desplegó las potenciales respuestas que debía dar el Estado al relator. Indicaba, en la presentación del documento, que:

6. Nos parece que la preocupación del Relato Especial (RE), conviene analizarla dentro de las acciones que normalmente éste realiza, en el ámbito de su mandato, cuando se presentan situaciones críticas en un país miembro. Este caso no tiene -por el momento- el alcance de la temática mapuche. [...]

8. Con esta lógica, sugeriríamos enviar una comunicación al RE, en la que se dé un mensaje político concreto, del compromiso del Gobierno del Presidente Sebastián Piñera, para abordar los problemas y desafíos de la comunidad Rapa Nui, con una perspectiva integral, a través de acciones concretas y visión de futuro (Oyarce, 2010).

Según el representante chileno, el gobierno había mantenido la línea directriz de proseguir con alternativas para el caso de la isla, destacando la mesa de trabajo expuesta anteriormente. Sostenía, por lo demás, que el Gobierno había regularizado las tierras de 26 familias, demostrando así una voluntad real de ayuda y cooperación hacia los pueblos origina- rios. No obstante, en este mismo borrador se minimizaban los daños a los rapanui, llegando a afirmar que solo tres de ellos habían sufrido heridas leves. La Comisión Interamericana de Derechos Humanos había evidenciado una situación diametralmente opuesta.

El 12 de enero de 2011, Anaya recordó al gobierno de Chile que, como signatario del Convenio 169 de la OIT y de otras normas internacionales relevantes, debía respetar el derecho de los "clanes rapanui sobre sus tierras ancestrales"30. La carta enviada al presidente Piñera por el International Indian Treaty Council (IITC) seguía la misma dirección. En esta misiva, Alberto Saldamando se refería a los desalojos de varios clanes, que seguían protestando por la alienación de sus tierras y territorios ancestrales. Reconocía la obra de James Anaya, pero observaba que el gobierno de Chile, pese a querer buscar un diálogo con los rapanui, recurría, más bien, al uso de la fuerza. Agregaba que el IITC notaba que Chile siempre había apoyado y votado los derechos indígenas. Por estas razones, y retomando la declaración de la ONU, los postulados del Comité de los Derechos Humanos y del CERD, Chile debía reconocer los derechos de los pueblos indígenas "a determinar su propio desarrollo y requieren que los Estados adquieran el consentimiento libre, previo e informado de los pueblos indígenas antes de que les sean impuestos proyectos de desarrollo"31.

El 6 de febrero, los desalojos prosiguieron y fue el Indian Law Resource Center (IRLC) ${ }^{32}$ el que se pronunció contra las acciones del gobierno. Frente a las violencias ejercidas, la CIDH pidió al IRLC que llevara a cabo una investigación de los últimos sucesos. Esta fue encabezada por su abogado Leonardo Crippa. Este último 
había recibido un informe detallado sobre las quejas del Parlamento Rapa Nui y sobre los abusos cometidos por el Estado chileno ${ }^{33}$. En este borrador, el Parlamento planteaba que Rapa Nui era una monarquía y que Chile caía en la ilegalidad cuando buscaba establecer su derecho en ese territorio.

El pensamiento de los autores de esta misiva retomaba, paso a paso, los diferentes argumentos de la OIT y de la Declaración de los Pueblos Indígenas. Se desprendía de él, en primer lugar, la exigencia de la desmilitarización de la isla. Se denunciaba que Chile la había usado para sus propios fines militares y que había cedido, también, a las presiones de Estados Unidos para reafirmar su poder durante la Guerra Fría. En segundo lugar, se acusaba a la CODEIPA de ser un instrumento del Estado de Chile para lograr sus objetivos colonialistas. Se ahondaba en que esta organización no representaba el principio de organización de los pueblos originarios según los convenios firmados. En tercer lugar, por estas faltas e incumplimientos, el Parlamento Rapa Nui pedía la descolonización. Sus miembros destacaban que "por reiteradas violaciones de los derechos humanos a los originarios de la Nación Rapa Nui, el Parlamento reafirma las aspiraciones de los jefes por la descolonización, porque Chile no merece tener la potestad sobre esta nación"34.

Con las violencias policiales acaecidas en la isla se dieron las pruebas necesarias para demostrar el carácter colonialista y opresor hacia el pueblo rapanui del Estado chileno. Es dentro de esta lógica que podía entenderse la denuncia entregada por Erity Teave a la Comisión del CERD en febrero de 2011. En ella relataba que los sucesos de violencias aún no se detenían. Citaba, por ejemplo, el desalojo del Parlamento
Rapa Nui en plena sesión y las persecuciones hacia el clan Hitorangi. La vocera incriminaba "la presión del prominente grupo económico de los Schiess y la injusticia colonialista del sistema gubernamental del gobierno de Chile"35. Vale la pena detenerse en la segunda parte de la acusación, a saber, la injusticia colonialista del sistema gubernamental. Si bien la vocera reconocía que estas denuncias escapaban del mandato de la Comisión del CERD, ahondaba en los temas urgentes para la situación de la isla:

1. La petición del Parlamento Rapa Nui a la Comisión del CERD era la desmilitarización y que se monitoreara el proceso.

2. La propuesta de negociar con Chile, el status quo ante velum, con la mediación de un país extranjero elegido por Rapa Nui.

3. La formación policial en Rapa Nui, a partir del entrenamiento y la capacitando de gente de la isla para tales cargos.

4. El control de la inmigración a la brevedad posible.

5. La negociación de un gobierno propio de acuerdo con la libre determinación, con base en las Resoluciones 1.514 y 1.541 del Charter de las Naciones Unidas de 1960.

Además de estas peticiones a la Comisión del CERD, el Parlamento se dirigió nuevamente al presidente Piñera el 21 de febrero. En esta carta (Carta del Parlamento dirigida al presidente Sebastián Piñera, 21 de febrero de 2011, Hanga Roa. APRN, 6582-6590), enviada con copia a los diversos organismos internacionales, la organización rapanui citaba el discurso del Ejecutivo en la ONU del 22 de septiembre de 
2010. Frente a la violencia continua por parte del Estado, esta organización informaba que se iba a presentar una demanda ante la Corte Interamericana de Derechos Humanos, para hacer valer sus derechos internacionalmente reconocidos, y también ante el Comité de Descolonización de las Naciones Unidades. En este mismo documento, el Parlamento explicitaba, de igual modo, una continuidad en la dimensión internacional de los diferentes tratados, fijando como punto de partida el de 1888. Los autores rapanui pedían el respeto al derecho inalienable a la libre determinación, que estaba ya en el "espíritu" del acuerdo de 1888 y que había sido posteriormente ratificado mediante la Declaración de la ONU sobre los Derechos de los Pueblos Indígenas, la Convención sobre la Discriminación Racial y el Convenio 169 de la OIT.

En ese mismo tenor, la Comisión del CERD se dirigió al gobierno chileno, un día después, para destacar, entre otros elementos, que los derechos indígenas en Chile no eran considerados en las reformas constitucionales. También llamaba a acelerar el proceso de restitución de las tierras indígenas y a detener las discriminaciones raciales hacia los pueblos originarios ${ }^{36}$. Estas demandas convergieron con la visita de Eni Faleomavaega a Rapa Nui el 21 de marzo. El congresista se reunió inicialmente en Santiago con Alfredo Moreno, ministro de Relaciones Exteriores; Felipe Kast, ministro de Planificación, y Sebastián Donoso, asesor especial para Asuntos Indígenas. En esta misión, Faleomavaega expresó que "En la actualidad, las personas y corporaciones no indígenas poseen la mayor parte de la tierra, y el gobierno chileno continúa favoreciendo a las empresas privadas interesadas en explotar la cultura Rapa Nui para obtener beneficios privados en lugar de restaurar la tierra a los Rapa Nui” (Faleomavaega, 2011).
Frente a estas presiones internacionales, el gobierno decidió intervenir en el décimo período de sesiones del Foro Permanente de Asuntos Indígenas de las Naciones Unidas el 20 de mayo de 2011. En esta presentación, el asesor especial para Asuntos Indígenas del gobierno de Chile, Sebastián Donoso, buscó mostrar la buena voluntad del gobierno chileno frente a las problemáticas de los pueblos originarios y en especial con el pueblo rapanui. En su tribuna, sostenía que el gobierno de Chile se encontraba iniciando una importante consulta sobre institucionalidad indígena según lo establecido por el Convenio 169 de la OIT y las diversas propuestas del relator especial Anaya. Esta consulta tocaba tres puntos clave, a saber:

1. El proyecto de reconocimiento constitucional de los pueblos indígenas.

2. Los proyectos de ley que crearán la Agencia de Desarrollo Indígena y el Consejo de Pueblos Indígenas, instituciones ambas que están llamadas a perfeccionar la institucionalidad de nuestro país en relación con los pueblos indígenas.

3. El procedimiento permanente que regule la participación y consulta establecida en el Convenio 169 de la OIT respecto a las medidas legislativas y administrativas susceptibles de afectarles directamente ${ }^{37}$.

Esta iniciativa, según el enviado chileno, se inscribía en uno de los cinco ejes de trabajo del Ejecutivo en materia de política indígena ${ }^{38}$. Por otra parte, el caso puntual de Rapa Nui implicaba un comentario especial. Citamos aquí: "Desde agosto de 2010, el gobierno ha impulsado un proceso de diálogo con el Pueblo Rapa Nui que aborda los principales temas de su preocupación: tierras, migración, estatuto 
especial y plan de desarrollo de la isla"39. Para llevar a cabo este proceso, las diferentes comisiones de trabajo estaban compuestas por distintos actores, como el Ejecutivo, las autoridades electas por la comunidad (integrantes de la CODEIPA y el Consejo Municipal) y por representantes de la sociedad civil de la isla.

Si bien esta presentación destacaba por su mutismo respecto de las violencias ejercidas, el asesor especial buscó demostrar que el Estado chileno sí ratificaba los convenios internacionales. Las continuas denuncias del Parlamento siguieron y trajeron a otra esfera el discurso de lucha contra las políticas del Ejecutivo. Si bien se apreciaba una continuidad en la tónica de las reivindicaciones rapanui, la influencia de los organismos internacionales obligó al Estado a semejantes presentaciones. Sin lugar a dudas, esta organización había logrado consolidar una estrategia dentro de la cual el Estado tenía que rendir cuentas a organismos de peso.

La directiva del Parlamento partió a comienzos del mes de julio de 2011a Ginebra para pedir a los expertos de Derechos Humanos una tribuna para dar a conocer las violencias ejercidas por el Estado y su inconsistencia. En un correo electrónico dirigido al relator especial Anaya, los miembros de esta organización presentaron la situación de la isla. En la misiva apelaban a los diferentes estatutos legales internacionales y señalaban que el estatus de Rapa Nui se regía por la imposición del Estado chileno, mismo que le negaba el derecho a la autodeterminación de su pueblo. Aprovechaban además para denunciar la falta de ética del gobierno de Chile respecto de las leyes internacionales:

La declaración oficial fue falsa con respecto a Rapa Nui. La misma declaración fue dada a la Corte Interamericana de Derechos Humanos, para evitar la medida cautelar, otorgada más tarde, en enero, para justificar los desalojos desproporcionados, brutales e ilegales de mi pueblo 40 .

En consecuencia, la vocera del Parlamento pedía una mediación entre las autoridades de Derechos Humanos y los expertos de la ONU y el gobierno chileno para reconocer: 1) La autodeterminación y el reconocimiento del reino Rapa Nui; 2) La desmilitarización urgente de la isla; 3) El reconocimiento de la constitución Rapa Nui; 3) La liberación de todas las tierras ancestrales para el pueblo Rapa Nui. Un par de días después, y tras reunirse con el relator, Teave volvió a referirse a estos puntos en una entrevista (Dufey, 2011), en la que denunció la subyugación y la ocupación del gobierno y el Estado chileno. En esa ocasión, la vocera planteó que urgía la apertura del diálogo de negociación y que debía renovarse el tratado.

Durante el Foro de las Naciones Unidas de 2011 se adoptó, entre los mecanismos de los expertos sobre los derechos de los pueblos indígenas, un tema clave que venía a respaldar las acciones del Parlamento. Se estableció así una mesa de trabajo para el desarrollo de un estudio sobre los pueblos indígenas y el derecho a participar en la adopción de decisiones ${ }^{41}$. El Parlamento participó activamente en estas mesas, lo que le dio la posibilidad de recibir una formación política internacional sobre la capacidad de agenciamiento de los pueblos originarios.

Por otra parte, en el mes de agosto, la ONG Indian Law, gracias a numerosas donaciones, envió un grupo de abogados para encontrarse con los líderes del Parlamento Rapa Nui. Tal como se indicó en el sitio de la ONG, por primera vez abogados indígenas especiali- 
zados en derechos humanos proveían información completa y acabada a pueblos originarios. Esta misión tenía como finalidad proteger a estos pueblos y darles las herramientas idóneas para resguardarse. Encabezadas por Robert Coulter, Leonardo Crippa y Senior Attorney, estas reuniones y diálogos giraron en torno a los derechos sobre las tierras y el derecho a la autodeterminación.

Por otra parte, el equipo de Indian Law se reunió con miembros del gobierno chileno para informarles de la voluntad del pueblo rapanui y abogar por mejores relaciones entre ellos. El equipo de abogados instaba al gobierno chileno a seguir ciertas recomendaciones e informarles, en un plazo de treinta días, sobre los siguientes puntos: 1) Información detallada sobre la alegada militarización de la isla y su efecto concreto, con ejemplos, sobre el pueblo rapanui en las diversas zonas de la isla; 2) Alegatos específicos relacionados a si el riesgo presunto se estaría dando para una comunidad o clan en particular o para todo el pueblo rapanui; 3) El estado actual del proceso de negociaciones entre el pueblo rapanui y las autoridades (Carta de Elizabeth Abi-Mershed, secretaria ejecutiva adjunta de la OEA dirigida a Leonardo Crippa, abogado de Indian Law, 19 de agosto de 2011, Washington, Estados Unidos, APRN, 6511-6513).

Lo postulado por este equipo tuvo profundos ecos en la Undécima Sesión del Foro de los Asuntos Indígenas de la ONU llevada a cabo en 2012. Celebrado entre el 7 y el 18 de mayo, el Foro presentó una serie de recomendaciones estrechamente relacionadas con los últimos sucesos ocurridos en Rapa Nui y en la Araucanía (Indígenas, 2012). La temática especial llamada "Doctrina del descubrimiento: su repercusión duradera en los pueblos indígenas y el derecho a recibir reparación por conquistas del pasado" inducía a un replanteamiento de los Estados colonizadores. Recordando el cuarto párrafo del preámbulo de la Declaración de las Naciones Unidas sobre los Derechos de los Pueblos Indígenas, el Foro Permanente condenaba las tretas jurídicas y políticas de los Estados colonizadores para desposeer a los pueblos indígenas de sus tierras. Una de estas, ya citada anteriormente, era la doctrina de la terra nullius, punto de partida de las reivindicaciones del pueblo rapanui. Estas manifestaciones debían ser entendidas, según estas recomendaciones, en el marco de la supuesta "supresión" de los derechos de los pueblos indígenas.

En esa misma ocasión se estableció una mesa de trabajo sobre la descolonización del Pacífico. En el estudio realizado en dicho espacio, Santi Hitorangi Atamu, en nombre de su clan y miembro del Parlamento Rapa Nui, entregó un primer esbozo del caso de la isla. En él sostenía que desde 1946 Chile no había asumido sus obligaciones y no había reconocido a Rapa Nui como un territorio no autónomo. Producto de esta situación, buscaba su admisión en la lista de los territorios no autónomos y unirse a las filas de los hermanos del Pacífico, a saber, Samoa, Polinesia francesa, Guam, Nueva Caledonia, Pitcairn y Tokelau, y trabajaba para crear las condiciones necesarias para la autodeterminación, en concordancia con la Resolución 1.514. En esta línea, proseguía, Chile había violado los mandatos de la Carta de la ONU, capítulo XI, y no había asumido sus responsabilidades para la administración de Rapa Nui. El Estado había fallado, entonces, en torno a dos ítems: con respecto a la cultura, las políticas económicas, sociales y educativas, y en desarrollar una política de autogobierno para Rapa $\mathrm{Nu}^{42}$. 
El informe intitulado "Estudio sobre la descolonización de la región del Pacífico", dirigido por Valmaine Toki, fue presentado en la sesión siguiente del Foro, en su duodécimo período. Según el balance de esta mesa de trabajo, se podía entender el fenómeno de la colonización como un asunto pendiente, pero que, a su vez, debía ser matizado:

\footnotetext{
La tendencia de la descolonización en el Pacífico no sigue el precedente sentado por Asia, África y el Caribe. El contexto temporal, el tamaño, la lejanía y la vulnerabilidad económica junto con [...] la determinación de algunas potencias coloniales en los territorios [...] proporcionan un telón de fondo único a la independencia y la descolonización del Pacífico. (Toki, 2013)
}

Además de citar a las diferentes potencias que seguían asentando su dominio en el Pacífico, se explicaba que permanecía una continua denegación de los derechos básicos establecidos en la Declaración de las Naciones Unidas sobre los Derechos de los Pueblos Indígenas. Para ejemplificar esta idea, Toki subrayaba que, en el caso de Rapa Nui, quienes reivindicaban de manera pacífica el respeto a sus derechos humanos eran calificados de terroristas (Toki, 2013). Cuando se constataban los daños causados por la colonización, los planteamientos del Parlamento por el derecho a la libre determinación cobraban nuevamente toda su pertinencia. Esta organización podía también ver en las poblaciones originarias de Nueva Caledonia, Polinesia francesa, Hawai'i y Papúa Occidental, que compartían las mismas aspiraciones, potenciales alianzas políticas para llevar a cabo su proyecto final. Estas alianzas se consolidaron en el Comité Especial para las solicitudes de independencia. Algunas solicitudes fueron exitosas, otras, como la del Parlamento Rapa Nui, no se han concretizado, pero siguen vigentes para sus miembros.

\section{Conclusión}

En este trabajo se expuso cómo el Parlamento Rapa Nui estableció conexiones consistentes con sus homólogos polinésicos. El primer acierto de esta institución fue establecer un lazo político, pero también identitario, con la Polinesia francesa, que ha sido desde larga data un referente para los rapanui. Junto a esta confirmación identitaria, el Parlamento ratificaba su propio concepto de nación. Tal como ha sido explicado aquí, las particularidades de Rapa Nui no se constriñen solo a la isla. En los últimos discursos de reivindicación del Parlamento, el territorio era entendido como la tierra ancestral, pero se le agregaba el espacio marítimo que lo rodea.

El segundo punto que se ha querido subrayar es la manera como el Parlamento pudo beneficiarse de un contexto favorable a los derechos de los pueblos originarios para introducir, en el espacio político, su anhelo más profundo, que es la autonomía. Amparándose en los tratados internacionales, la falta de representatividad política y el carácter particular de la cultura insular, la noción de autonomía fue declarada como válida por el Parlamento. Para hacer realizable este designio era importante fortalecer los lazos entre las naciones independientes del Pacífico. Así, frente a un posible fallo negativo con respecto a la demanda judicial en contra del Estado chileno, la estrategia de la institución rapanui fue la de recurrir a la Corte Internacional de La Haya. Para que esta sea formalizada: "Rapa Nui necesitará el respaldo político y lobby de las Naciones Independientes del Pacífico, cuyo status internacional son miembros de las Naciones Unidas, para presentar nuestra causa ante la corte de la Haya"43. 
Finalmente, cabe destacar que la fuerza política y discursiva del Parlamento Rapa Nui ha ido disminuyendo desde el año 2015. Existen diferentes explicaciones a esta tendencia. La primera es la aparición de otras instituciones locales, como el Hōnui, que tomó en cuenta a los 36 representantes de los jefes de familias rapanui. El Hōnui ratificaba su derecho inalienable de administrar sus recursos naturales y culturales, como, por ejemplo, la administración del Parque Nacional. Desde esta nueva organización se favoreció e impulsó la constitución de Mau Henua como garante del espacio terrestre y marino. En la actualidad, Mau Henua administra, en conjunto con la Corporación Nacional Forestal (CONAF), el Parque. Una segunda razón es que el Parlamento ha buscado privilegiar el crecimiento de ambas organizaciones para poder, progresivamente, apelar a una autonomía real en la isla. Estas nuevas instancias no han dejado indiferentes a los miembros de la comunidad, quienes se han dividido respecto del modelo de gestión a seguir para las décadas venideras.

Una tercera explicación, y tal como pasó con otras instituciones, como el Consejo de Ancianos, el peso carismático de los líderes ha provocado una difícil capacidad de sucesión y renovación de sus líderes. Las nuevas generaciones rapanui tendrán que buscar otras terminologías discursivas e instancias para hacer valer sus derechos. Pero resulta extremadamente interesante ver que organizaciones internacionales pueden ser fuentes de inspiración y cooperación para los rapanui del siglo XXI.

\section{Notas}

\footnotetext{
1 Juan Chávez, fundador del Parlamento Rapa Nui y líder del otrora Consejo de Ancianos, presentó el Consejo № 2 al Congreso Nacional el día 4 de septiembre de 1994, lo que puso en evidencia que tanto el Parlamento como el Consejo de Ancianos existían como instituciones políticas, previas a la llegada de Chile como Estado colonizador (Misiva de Juan Chávez a la Cámara de Diputados, 4 de septiembre de 1994, Hanga Roa. Archivo Alfredo Tuki, 4300-4303). El Parlamento provenía, entonces, de una clara tradición política rapanui, que remontaba al período del rey Atamu Tekena. Este último, proclamado por una parte de la comunidad católica rapanui, tenía un consejo compuesto por los diversos representantes de cada clan de la isla. Con la incorporación a Chile el 9 de septiembre de 1888 , el rey quiso mantener una estructura de autoridad rapanui. Es importante señalar que en este texto se utiliza "Parlamento" para hacer referencia al nombre de esta organización y en ningún caso para referirse al poder legislativo chileno

${ }^{2}$ El Consejo № 2 apareció en 1994 tras las diferencias irreconciliables entre su presidente, Alberto Hotus, y su vicepresidente, Juan Chávez. Durante la dictadura habían conformado el Consejo de Ancianos. Este era, según Hotus "una entidad de análisis y toma de decisión, formada por 36 personas que representan cada apellido de la isla, cuyo objetivo es defender nuestros derechos a la territorialidad, cultura e intereses de Rapa Nui" (Hotus, 1988, p. 351). Posteriormente, con la vuelta de la democracia, se enrostró
}

a Hotus su acumulación de cargos en la isla y su acercamiento a la Concertación. Estas tensiones persistieron hasta 1998, fecha en la cual este ganó las elecciones de la CODEIPA y donde "el Consejo № 2 había quedado virtualmente disuelto" (Delsing, 2010, p. 272).

${ }^{3}$ El contexto era particularmente complejo en la isla, Delsing (2010) entrega luces de los acontecimientos que desembocaron en la fundación del Parlamento. En el mes de julio de 2001, dos casas pertenecientes a Erity e Inés Teave, y situadas en terrenos del Parque Nacional, fueron destruidas por una excavadora en presencia del gobernador y miembros de la CODEIPA. Las casas construidas se encontraban en un territorio ancestral, según las hermanas Teave, y por el derecho relacionado dicho espacio les correspondía.

${ }^{4}$ Foerster y Montecino (2016) han buscado complejizar el aporte del Estado y de sus representantes en la isla. Para ellos, los "agentes imperiales" presentan numerosas aristas que permiten lecturas alternativas de la situación en Rapa Nui. La matriz teórica de estos autores recupera una triangulación dinámica entre los ejes siguientes: Estado, comunidad y la Compañía Explotadora de Isla de Pascua (CEDIP).

${ }^{5}$ Hilda Lini, jefa (motarilavoa) de la nación Turaga de la Isla de Pentecostés en Vanuatu, ayudó a su nación a obtener su independencia en 1980. Después de su servicio público, fue directora del Pacific Concerns Resource Centre y representante de la región del Pacífico en la Conferencia de Revisión de No Proliferación de Armas Nucleares de las Naciones Unidas. 
${ }^{6}$ Reporte Regional del Pacífico presentado por la Motarilavoa Hilda Lini, directora del Pacific Concerns Resource Centre, en la Tercera Sesión del Foro Indígena, ONU, 10-21 mayo de 2004. Archivo Parlamento Rapa Nui (APRN), 6274-6285.

7 Ídem.

${ }^{8}$ Carta de Juan Chávez Haoa, presidente del Parlamento, dirigida al Gobernador Provincial de Isla de Pascua, 3 de noviembre de 2003, Hanga Roa. Archivo Alfredo Tuki, 4279-4286.

${ }^{9}$ Correo electrónico enviado por Erity Teave a las diversas agrupaciones indígenas polinesias y a organizaciones de la ONU, 16 de marzo de 2004, Hanga Roa. APRN, 6260.

${ }^{10}$ Fax enviado por el Parlamento Rapa Nui a Rex Rumakiek, director asistente de la oficina de descolonización en Fidji, 22 de julio de 2007, Hanga Roa. APRN, 6267.

11 Íbidem.

${ }^{12}$ Carta de Erity Teave dirigida al presidente y delegados del Comité de Descolonización de Naciones Unidas, 19 de mayo de 2008. APRN, 6599-6600.

${ }^{13}$ PIANGO está radicada en Fidji y fue fundada hace 25 años. Se trata de la principal organización no gubernamental regional, pues considera a los 23 países y territorios de las islas del Pacífico. En la actualidad busca establecer una dirección dinámica para vigorizar la sociedad civil basándose en la comprensión del contexto del Pacífico.

${ }^{14} \mathrm{Carta}$ de Leviante Araki a la gobernadora $\mathrm{C}$. Hotus para informar sobre la manifestación pacífica, 20 de mayo de 2008, Hanga Roa. APRN, 6581.

15 Ídem.

${ }^{16}$ El Foro fue establecido el 28 de julio de 2000 por la Resolución 2.000/22, con el mandato de examinar las cuestiones indígenas en el contexto de las atribuciones del Consejo Económico y Social (ECOSOC) de la ONU relativas al desarrollo económico y social, la cultura, el medio ambiente, la educación, la salud y los derechos humanos.

17 Intervención de Erity Teave en el Foro Permanente de las Naciones Unidas, Ítem Derechos Humanos donde presenta al Parlamento, 10-21 de mayo de 2004. APRN, 6591.

${ }^{18}$ Esta sesión tuvo lugar entre el 21 de abril y el 2 de mayo de 2008 en la sede de las Naciones Unidas en Nueva York. La temática fue: "El cambio climático, la diversidad biocultural y los medios de vida: la custodia por los pueblos indígenas y nuevos retos".

${ }^{19}$ Séptima Sesión de las Naciones Unidas Foro Permanente para Cuestiones Indígenas, abril de 2008. APRN, 6241-6242.

20 Ídem.

${ }^{21}$ Octava Sesión de las Naciones Unidas, Foro Permanente de Asuntos Indígenas, mayo de 2009. APRN, 6243-6246.

${ }^{22}$ Carta del Parlamento dirigida a la presidenta Michelle Bachelet, 30 de julio de 2009, Hanga Roa. APRN, 6628.

${ }^{23}$ Carta del Parlamento dirigida a Patricio Rosende, subsecretario del Interior, 21 de octubre de 2009, Hanga Roa. APRN, 6629.

${ }^{24}$ Carta del Parlamento dirigida a la presidenta Michelle Bachelet, op. cit.

${ }^{25}$ Carta del Parlamento dirigida a Patricio Rosende..., op. cit.

${ }^{26}$ Carta del Parlamento Rapa Nui al presidente Sebastián Piñera, 6 de abril de 2010, Hanga Roa. APRN, 6757-6758.

${ }^{27}$ Carta dirigida a Carlos Llancaqueo, Comisionado Presidencial para
Isla de Pascua, 8 de junio de 2010, Hanga Roa. APRN, 6700-6703.

${ }^{28}$ Carta del Parlamento dirigida al presidente Piñera, 30 de agosto de 2010, Hanga Roa. APRN, 6619-6622.

${ }^{29}$ Carta del Parlamento firmada por Leviante Araki, Erity Teave y Johny Tuki, dirigida al presidente Sebastián Piñera, con copia a la ONG Indian Law, 20 de noviembre de 2010, Hanga Roa. APRN, 6762-6763.

${ }^{30}$ Declaración del Relator Especial de la ONU sobre los derechos de los pueblos indígenas, James Anaya, ante los desalojos de indígenas rapanui, 12 de enero de 2011. APRN, 6696.

${ }^{31}$ Carta del International Indian Treaty Council al presidente Sebastián Piñera, sobre los graves asuntos de los pueblos indígenas en Rapa Nui, firmada por Alberto Saldamando, 20 de enero de 2011. APRN, 6286-6287.

${ }^{32}$ The Indian Law Resource Center es una organización sin fines de lucro de abogados establecidos y dirigidos por amerindios. Esta agrupación provee asistencia legal sin cargos a los indígenas y nativos de Alaska que trabajan para proteger sus tierras, recursos, derechos humanos, medioambiente y herencia cultural. Su misión principal es proteger el bienestar de los indígenas y otras naciones tribales y tribus (www.indianlaw.org).

${ }^{33}$ Borrador para Leo Crippa sobre la situación de la Isla, 3 de enero de 2011. APRN, 6607-6608.

34 Ídem.

${ }^{35}$ Comisión CERD, firmado por Erity Teave, 15 de febrero de 2011. APRN, 6691-6693

${ }^{36}$ International Indian Treaty Council, Rapa Nui complaint and an appeal for urgent action to the CERD Committee by Alberto Saldamando, 22 de febrero de 2011. APRN, 6687-6690.

${ }^{37}$ Discurso de Sebastián Donoso, asesor especial para Asuntos Indígenas del gobierno de Chile, ante el Foro Permanente para las Cuestiones Indígenas de Naciones Unidas, 20 de mayo de 2011. APRN, 6785-6788.

${ }^{38}$ Se recuerdan aquí los cinco ejes de trabajo propuestos por la administración del presidente Piñera: 1) Fomentar decididamente las culturas e identidades indígenas; 2) Corregir y mejorar los procedimientos de entrega de tierras, asociándolos a iniciativas de apoyo productivo;3) Reestructurar la institucionalidad vigente; 4) Continuar con la instalación de procesos efectivos de consulta y participación indígena y 5) Políticas focalizadas hacia los pueblos indígenas.

${ }^{39}$ Discurso de Sebastián Donoso, op. cit.

${ }^{40}$ Carta de Erity Teave a James Anaya, relator especial de los Derechos Humanos, 10 de julio de 2011, Hanga Roa. APRN, 6296-6299.

${ }^{41}$ Asamblea Consejo de Derechos Humanos, mecanismo de expertos sobre los derechos de los pueblos indígenas, 11-15 de julio de 2011. APRN, 6805-6808.

42 Ítem 8: Futuros trabajos en el Foro Industria y Estudios sobre Descolonización en la región del Pacífico. Introducido por Santi Hitorangi Atamu, 20-31 de mayo de 2012. Archivo Teave, 6342-6343.

${ }^{43}$ Solicitud de Leviante Araki de reunión con el relator especial Don James Anaya con el objetivo de informar del primer encuentro de los Parlamentos de la Polinesia, recientemente constituido en 2013, 14 de abril de 2014. APRN, 6658-6660. 


\section{Referencias bibliográficas}

Anaya, J. (2009). Promoción y protección de todos los derechos humanos, civiles, políticos, económicos, sociales y culturales, incluido el derecho al desarrollo. Consejo de Derechos Humanos. Recuperado de http://www.acnur.org/fileadmin/Documentos/BDL/2010/8057. pdf?view $=1$

(2011). Comunicación relator indígena Isla de Pascua. 9 de diciembre. Misión Ginebra 2001, Vol. 1, EO1 302, № 1161. Ginebra.

Bajtín, M. (2005). Problemas de la poética de Dostoievski. México: Fondo de Cultura Económica.

Cooperativa (2010a). Piñera ante la ONU, no hemos dado a los pueblos originarios las oportunidades que merecen. 23 de septiembre. (2010b). Abogado de clanes rapanui acusa absoluto silencio del gobierno ante la CIDH. 7 de diciembre.

Delsing, R. (2010). Articulating Rapa Nui: Polynesian cultural politics in a Latin American Nation-State. Santa Cruz: University of California.

(2017). Articulando Rapa Nui: Políticas culturales polinésicas frente al Estado chileno. Santiago: LOM.

Dijk, T. van (1999). El análisis crítico del discurso. Anthropos (186), 23-36.

Dufey, A. (2011). Entrevista con Erity Teave (Rapa Nui), Rapa Nui pide autonomía. 14 de julio. Swisslatin. Ginebra.

Faleomavaega, E. (2011). Congressman Faleomavaega to Visit Rapa Nui. 21 de marzo. Indian Law Resource Center. Recuperado de https://indianlaw.org/rapa-nui/congressman-faleomavaega-visitrapa-nui.

Foerster, R. \& Montecino, S. (2016). A 100 años de la rebelión de Angata: ¿Resistencia religiosa o secular? Las complicidades Tire y los múltiples sentidos de la revuelta de 1914 en Rapa Nui. Chungará, 48(1), 91-101.
Friedman, J. (2003). Globalizing languages: Ideologies and realities of the contemporary global system. American Anthropologist, 105(4), 744-752.

Hotus, A. (1988). Te Mau Hatu'O Rapa Nui: Los soberanos de Rapa Nui, pasado, presente y futuro. Santiago: Emisión.

Indígenas, F. P. (2012). Informe sobre el 11ํperíodo de sesiones. E/C.19/2012/13. Nueva York.

Letelier, J. P. (2010). Informe preliminar sobre los incidentes ocurridos el día 3 de diciembre y visita inspectiva a Rapa Nui. Asociación Latinoamericana para los Derechos Humanos.

Muñoz, D. (2017). Diaspora Rapanui (1871-2015). L'île de Pâques, le Chili continental et la Polynésie française: Une ethnographie historique de la mobilité dans une société transnationale. (Tesis inédita de doctorado en antropología social y etnología). École des Hautes Études en Sciences Sociales, París.

Oyarce, P. (2010). Carta dirigida a SEPREREP, Tema Isla de Pascua. 14 de diciembre. Misión Ginebra 2010, Vol. 1, EO1 302, № 1168 .

Rongo Rapa Nui, G. P. (2003). Informativo Rapa Nui. Rongo Rapa Nui (10), 1-4.

Smith, L. T. (2012). Decolonizing methodologies, research and indigenous peoples. Londres: Zed Books.

Stavenhagen, R. (2003). Informe del Relator Especial sobre la situación de los derechos humanos. Organización de las Naciones Unidas, Comisión de Derechos Humanos. Recuperado de https:// documents-dds-ny.un.org/doc/UNDOC/GEN/G03/170/94/PDF/ G0317094.pdf?OpenElement.

Toki, V. (2013). Estudio sobre la descolonización de la región del Pacífico, Naciones Unidas. 19 de diciembre. E/C.19/2013/12. 